\title{
Magnetically-Channeled SIEC Array (MCSA) Fusion Device for Interplanetary Missions
}

\author{
G. H. Miley, R. Stubbers, J. Webber, H. Momota \\ University of Illinois, U-C,Department of Nuclear, Plasma and Radiological Engineering \\ 103 S. Goodwin Ave., Urbana, IL 61801 USA \\ (217)333-3772,ghmiley@uiuc.edu
}

\begin{abstract}
A radical new Inertial Electrostatic Confinement (IEC) concept, the Magnetically-Channeled Spherical-IEC Array (MCSA) fusion propulsion system, was proposed earlier for use in the high performance Space Ship II fusion propulsion ship (Burton, 2003). This ship was designed for a fast manned round trip mission to Jupiter. The MCSA fusion power plant represents a key enabling technology needed for this mission. The details of the proposed MCSA design are presented here, along with a discussion of some possible experiments that could be performed to confirm key physics aspects.
\end{abstract}

\section{INTRODUCTION}

A radical new Inertial Electrostatic Confinement (IEC) concept, the Magnetically-Channeled Spherical-IEC Array (MCSA) fusion propulsion system is proposed for interplanetary space travel. IEC fusion concepts are widely recognized to be attractive for space power because they are simple and lightweight. However, existing experimental IEC concepts, while very successful for low-level power neutron sources, do not project to high power space applications due to poor confinement time scaling and grid heating/losses. The MCSA concept addresses both issues - eliminating the need for a central grid and providing improved confinement time scaling. While the geometry uses coupled Spherical-IEC (SIEC) units, the real innovation in the MCSA concept lies in the formation of potential and magnetic field surfaces that retrap leaking particles while forming a gridless potential well confinement region. This results in a greatly improved fuel economy and significantly improves the overall confinement time. Because of the non-Maxwellian nature of SIEC confinement, aneutronic fuels, such as D- ${ }^{3} \mathrm{He}$, can be easily implemented. These fuels provide an ample source of high-energy protons (or alphas) to neutron ratio. It requires no massive blanket and provides very high efficiency along with the application of a Traveling Wave Direct Energy Convertory or TWDEC (Momota, 1999). Thus, the MCSA concept has the enabling potential for the requirements of future deep space mission by providing a very high power-density propulsion system. This premise was amply demonstrated in a conceptual design study by Burton et al. (2003) which used a MCSA to accomplish a fast manned mission to Jupiter. In the present paper, we discuss the basic MCSA concept and pin point some physics issues that must be resolved to access the feasibility of this concept. Some important supporting data carries over from prior SIEC experiments, but a full MCSA configuration has yet to be studied experimentally. If proven feasible, the MCSA development path would involve experiments at progressively higher powers aimed at the ultimate demonstration of a full-scale multi-MW propulsion unit. Some thoughts about possible next step experiments are presented.

\section{MAGNETICALLY-CHANNELED SIEC ARRAY (MCSA) CONCEPT}

The SIEC device represents a very attractive approach to fusion power due to its' high power density combined with a simple lightweight structure. However, the need for an internal grid and a limited confinement time has prompted several researchers to seek an alternative configuration that combines the SIEC and magnetic fields. Thus, R. W. Bussard proposed a hexapole field configuration to confine electrons while ions remain electrostatically confined. 
(Bussard, 1991) Large electron losses through the field cusps remain an issue for this concept, however. D. Barnes and R. Nebel have proposed a Penning trap concept (Barnes, 1998). This approach however is limited to use of a large number of very small diameter devices due to the Brillouin density limit. Most recently, University of Illinois researchers recently proposed the Magnetically-Channeled SIEC Array (MCSA) concept for a hybrid magnetic assisted SIEC configuration that addresses the issues faced in these earlier hybrid concepts.

The MCSA is illustrated conceptually in Figure 1. The SIEC is confined in a hexapole field configuration, quite different from the hexapole field proposed by R.W. Bussard (1991), that is in turn located in a field channel $\left(\mathrm{B}_{\mathrm{z}^{-}}\right.$ field) created by a column of Helmholtz coils. The field strengths of these coil sets is adjusted such that the fields cancel in the center of the SIEC, giving a larger field null region compared to that in a cusp confined field. The present configuration retains the advantage of stability due to good field curvature obtained in a cusp, plus it also effectively closes the "belt" loss cone as shown later in, leakage in that direction is led around the hexapole coils and back into the confinement region, termed here as "recirculation." Losses, due to scattering into the spindal loss cone along the z-axis, including stochastic scattering due to the violation of adiabatic invariance in the field null region, still occur without the collisionallity required in "linked Mirrors" eonfiguration. However, with the present channel configuration, the axial losses from one SIEC configuration enter a neighboring unit. Thus, as they pass through the field null region in that unit, stochastic scattering leads to "retrapping" of much of this flow. Experimental verification of this "retrapping" is then a second physics Proof-of-Principle (POP) objective. In this fashion, an array of multiple SIECs increases the overall confinement time roughly in proportion to the number of units. In operation, electrons would be magnetically confined as described, providing electrostatic confinement of ions injected into the SIEC region. An additional benefit of this configuration is that in a reactor embodiment, both leaking fuel plasma and energetic charged fusion products (e.g. the $14-\mathrm{MeV}$ proton from $\mathrm{D}-{ }^{3} \mathrm{He}$ ) can be collimated and aimed into a direct energy converter such as Traveling Wave Direct Energy Converter, TWDEC (Momota, 2000; Momota, 1992; Momota, 1999). This results in a high overall energy conversion efficiency. Alternately, for space propulsion, the proton beam, augmented by injection of heavy atoms, can be directly exhausted for thrust.

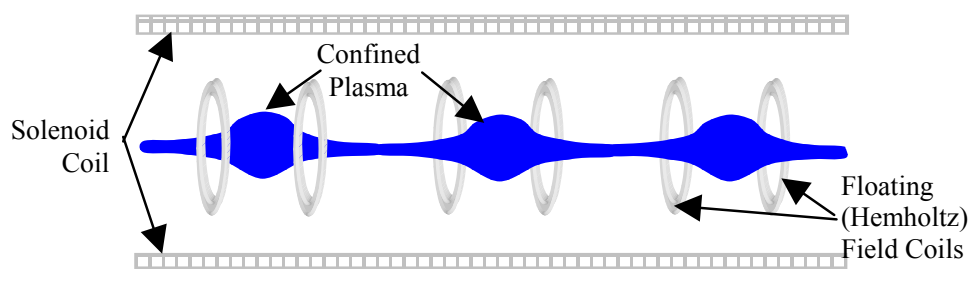

FIGURE 1. Illustration of a Three-Unit MCSA Device.

If the physics of this concept were found to be valid, a very attractive power plant would be possible. This was vividly demonstrated in the Space Ship II, study where a D- ${ }^{3} \mathrm{He}$ fueled MCSA was combined with a TWDEC and ion gun to provide a propulsion unit for deep space missions. (Burton, 2003) This design provided the thrust and specific power needed for a manned round trip to Jupiter in about 1 year, a target set by NASA due to Space radiation effect build up on Astronauts for longer trip times. While the added magnets add more complexity than for a "pure" SIEC, as shown in this design study, their size and weight were still minimal compared to conventional magnetically confined fusion devices such as a Tokamak. While weight per se is not so crucial for land-based electrical power plants, these aspects are in effect reflected in reduced costs and easier maintenance. The combination of D- ${ }^{3} \mathrm{He}$ fuel and TWDEC resulted in a high conversion efficiency $(>60 \%)$ and reduced weight (cost) for the energy conversion subsystem (Burton, 2003). While the MCSA concept leads, in principle, to a very attractive power unit, several key new physics aspects of the concept (namely "recirculation" and retrapping") need to be verified experimentally. One possible approach to a next step experiment would involve an electron-based simulation experiment. This would be discussed later. 


\section{HEXA-POLE MAGNETIC FIELD ASSISTED SIEC}

An inertial force is able to confine a low-pressure SIEC plasma. However, as the plasma pressure increases, magnetic assistance becomes important. In the MCSA configuration, the SIEC plasma expands outwards to the region of strong magnetic field formed by the outer magnetic channel. Thus, a high-pressure plasma is automatically confined by the magnetic field in the MCSA configuration.

A brief discussion of particle loss mechanisms is instructive. A particle that travels along the z-axis can escape from the confinement region. The strong magnetic field outside the confinement region, however, reflects particles that satisfy the following condition:

$$
v_{z}^{2}>\left(\frac{B_{m}}{B^{(j)}}-1\right) v_{\perp}^{2}-\frac{2 q_{i}}{M_{i}} \phi
$$

The notations here are traditional except that $B_{m}$ is the maximum magnetic field along z-axis and $B^{(j)}$ is the minimum magnetic field in the region where the $\mathrm{j}$-th particle conserves magnetic moment. Electrons have a smaller loss-cone angle, resulting, therefore, in smaller losses until potential fields form to equalize ion-electron loss rate. The loss flux for $\mathrm{j}$-th species of particles can be obtained as:

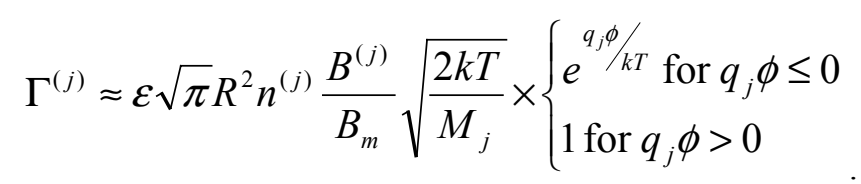

The steady state condition: $e \Gamma^{(D)}+2 e \Gamma^{(3 H e)}=e \Gamma^{(e)}$ along with the expression of loss fluxes gives a plasma potential of the form:

$$
e^{e \phi / k T}=\frac{1}{3 \sqrt{2}} \frac{B^{(D)}}{B^{(e)}} \sqrt{\frac{m}{M_{p}}\left(1+2 \frac{B^{\left.{ }^{3} H e\right)}}{B^{(D)}}\right)} .
$$

In a MCSA, the effective mirror ratio is naturally very large, e.g. $B_{m} / B^{(D)} \sim 100$, for the deuterium ions and other species have even larger mirror ratios. The large mirror ratio of this high-pressure MCSA fusion unit gives a large confinement time, which can be further improved by forming a multi-unit configuration. For example, in the Space Ship II study, a plasma potential of $-0.405(\mathrm{kT} / \mathrm{e}) \mathrm{V}$ and a particle confinement time of $\sim 20 \mathrm{~ms}$ were predicted for a representative D- ${ }^{3} \mathrm{He}$ SIEC fusion unit in a 20 unit multiple array.

\section{SCALING LAWS}

As previously discussed, the SIEC is non-Maxwellian in nature. However, as a very conservative, first look at scaling, the assumption of a Maxwellian distribution provides a conservative low-order approximation for the scaling. As discussed earlier, the mirror loss cones in an MCSA device should be over a small loss region in velocity space, resulting in a nearly Maxwellian distribution function in the confined region of velocity space. The loss-rate, $\Gamma^{(j)}$, of the $j$-th particle species in an MCSA, assuming a Maxwellian distribution, is given by Equation (2) for a single MCSA plasma unit.

The geometrical factor, $\varepsilon$, typically takes on a value of $\sim 4 \times 10^{-3}$. The confinement time is proportional to the volume of the plasma divided by the loss flux. Because the loss rate scales as $\mathrm{R}^{2}$, and the plasma volume scales as $\mathrm{R}^{3}$, the particle confinement time should scale linearly with the radius $\mathrm{R}$ for the unit. The confinement time should also scale linearly with the number of units, $\mathrm{N}$, in the MCSA, so the overall confinement time becomes: 


$$
\tau^{(j)} \propto R \times N .
$$

\section{HIGH POWER MCSA PHYSICS ISSUES}

For the purpose of achieving a practical SIEC fusion power unit, a plasma pressure must be contained that is much higher than that so far obtained with inertial and electrostatic forces alone. Fortunately, in a MCSA, a strong magnetic field (e.g. $\sim 6 T$ ) can surround the SIEC plasma. Such a configuration would have a relatively simple, linear, axi-symmetric geometry. It resembles ion many ways a series of coupled multiple cusp confinement devices. Unlike simple cusps, the present field configuration avoids the large losses from the "belt" loss cone that hamper a conventional cusp. This is achieved because the fields are formed such that leaking particles recirculate back into the confinement column. In addition, leakage out of the line cusp is stochastically scattered and retrapped in the neighboring SIEC unit. The high-density SIEC plasma is confined magnetically in the region of the Helmholtz fields. The diamagnetic current in the high-pressure plasma will help extend the magnetic field region out to the strong channel field. Fuel plasma particles would be confined by the magnetic field except for those traveling along the field line near the axis. Since the magnetic field approaches zero at the center of the core region, particles traveling along the axis into the region have no conserving magnetic moment, i.e., they suffer from collision less pitch-angle scattering (the KAM process or Stochasticity) at every passage through the central region. Takahashi et al. (1997) has previously studied the loss time of $\mathrm{D}-{ }^{3} \mathrm{He}$ fuel particles confined in a field reversed geometry. They found that the KAM process takes the place of the collision time of ions trapped in a mirror confinement and devised an approximate expression for the KAM dominated confinement time. This estimate has been employed here to evaluate the performance of the present MCSA configuration theory. Due to the ambipolar potential and extremely large effective mirror ratio along the magnetic axis, the estimated plasma confinement time in a $1 \mathrm{~m}$ radius SIEC unit approached $\sim 20 \mathrm{~ms}$. Since the confinement time is proportional to the linear scale, (i.e. to the number of units connected in a series), a series of 25 units could give a confinement time of $\sim 1 \mathrm{~s}$. The value is close to the goal desired for a power plant. However, experimental verification of the basic physics involved in the MCSA concept is essential before its future as a power plant can be fairly evaluated.

\section{EXPERIMENTAL VERIFICATION OF CONCEPT PHYSICS}

The addition of magnetic fields in the MCSA should eventually allow confinement of a high-pressure reactor-grade fusion plasma. Given the ability to confine fusion-grade plasma, the remaining parameter for breakeven fusion is the confinement time. The objective of next step experiments would be is to test/prove the physics principles of the MCSA concept. The two key physics issues are (i) the ability of the MCSA to exploit KAM processes to provide re-trapping of axial-loss particles in neighboring units and (ii) the ability of radial-loss particles, "belt-losses", to recirculate back into the confinement region along lines of force. These two phenomena and possible experimental verification experiments will be discussed next.

\section{RECIRCULATION OF RADIAL BELT CONE LOSSES}

The field configuration and a hypothetical particle trajectory through a belt cusp are shown in Figure 2.

Because the magnetic field lines that exit the cusp-like field reconnect to the confinement region within the coils, plasma particles that exit through the belt cusp loss cone will recirculate back into the confinement region. This recirculation significantly increases the confinement time verses that of a simple cusp devise, leading to a favorable concept. The linear scaling of an MCSA fusion device with plasma radius, as well as successful operations of multiple units in the array, are in large part controlled by this recirculation effect. Hence, demonstration of the recirculation effect becomes a key for confirming the physics of the MCSA. 


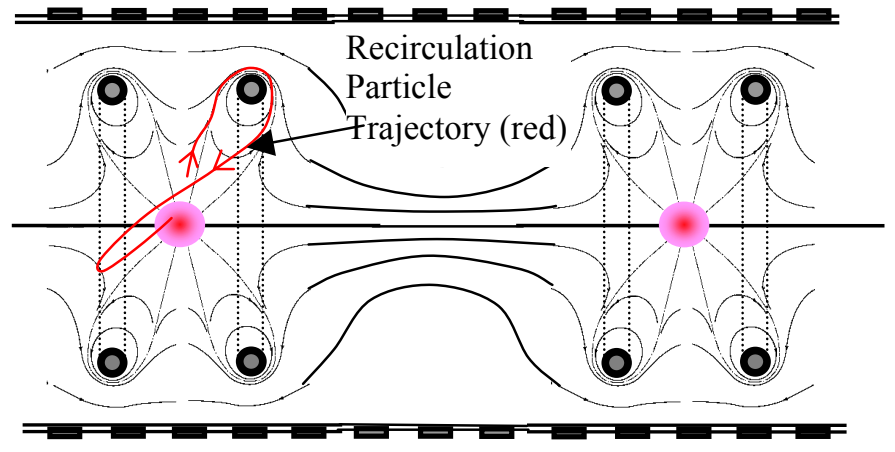

FIGURE 2. Diagram of Belt-Cusp Fields and Particle Recirculation.

The recirculation study could be done in stages, progressively introducing more complex plasma sources in the SIEC region. As shown in Figure 3, a filament type electron gun source would be located on axis such that an electron flux flows into the left SIEC unit where KAM scattering traps a fraction of the electrons in the unit. For initial studies in the first year, an electron gun will serve as a source and electron flow will be studied. Verification of recirculation characteristics could be carried out with three probes. The first probe would be a circular-ribbon collector plate (Faraday cup) mounted coaxially with the coils and near one of the inner coils. Another collector plate would be placed at one end of the coil array. The third probe, a Langmuir probe for measuring density, is placed in the confinement region on a movable feedthrough. The confinement coils, electron gun and probe arrangement for these experiments are shown in Figure 3.

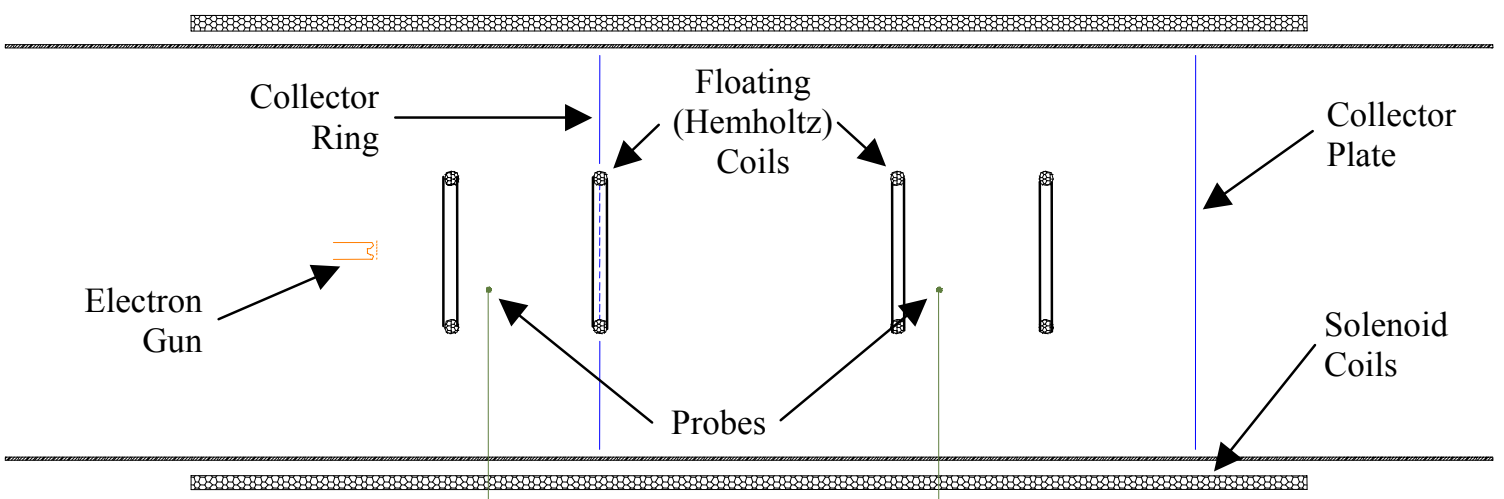

FIGURE 3. Coil, Electron Gun and Probe Arrangement for E-Beam Test with Ring Collector.

Verification of the recirculation effect would be done by first running the device with the left set of Helmholtz coils operating and with the collector ring and the collector plates installed. The electron density and currents to both plates would be measured. The ribbon collector plate would then be removed and measurements would be repeated. The current ratio observed ( $2^{\text {nd }}$ unit off and on) provides a test for the theoretical model of recirculation. The density ratio would provide data relating to the confinement time improvement caused by recirculation of the belt losses.

To extend the experiments to a plasma, a plasma gun could ultimately be used in place of the electron source. Using a plasma instead of only an electron source provides a better simulation of an actual fusion-plasma device because both charge species are present, allowing amipolar flow effects. As bulk RF generated plasma could be used as an intermediate step (before installing the gun) to initiate plasma experiments, but this approach requires a relatively higher background gas pressure. Still it could provide important intermedediate information regarding the confinement characteristics of plasma in an MCSA, and the effect of neutral particle contamination. 


\section{RETRAPPING OF AXIAL-LOSS PARTICLES}

Axial plasma particle loss escaping out through the end cone (spindle cone) of the first MCSA unit should be retrapped in the second unit because of the KAM effective scattering. This KAM scattering occurs as particles move into the null-field region within each SIEC unit. Figure 4 illustrates the axial magnetic field component along the centerline of the two-unit MCSA experiment. Particles escaping from the low field region of an MCSA unit into its neighboring unit pass through the high magnetic field region between the two units. Upon entering the SIEC region, the direction of the particle is effectively randomized when it losses adiabatic invariance during passage through the field null region. Although those particles with high velocities parallel to the magnetic field, $\mathrm{v}_{\|}$, (relative to their perpendicular velocity, $\mathrm{v}_{\perp}$ ) will not experience as much effective scattering, many of the particles entering the nullfield region should experience enough scattering to be retrapped.

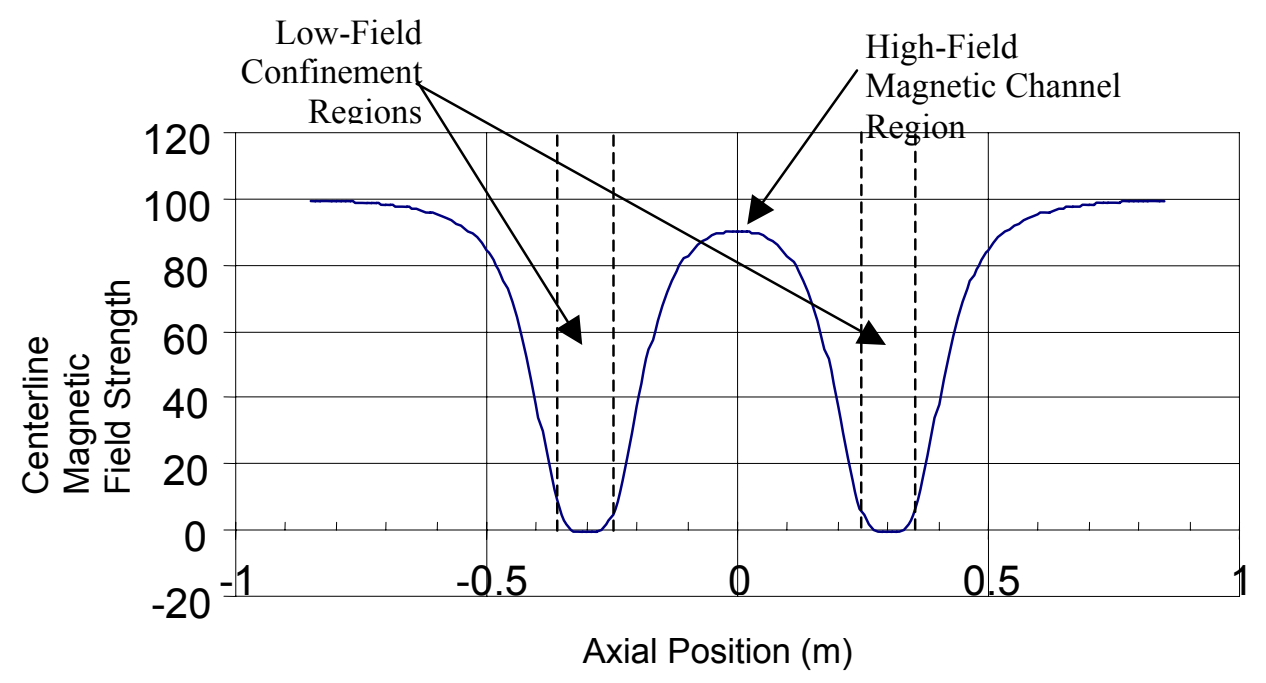

FIGURE 4. Axial Magnetic Field Strength Along Two-Unit MCSA Centerline.

Figure 5 illustrates this KAM scattering process conceptually. Before entering the null-field region, particles have a gyroradius that depends on their perpendicular velocity, $\mathrm{v}_{\perp}$. If $\mathrm{v}_{\perp}$ is large compared to $\mathrm{v}_{\|}$, the effective scattering angle will be large, resulting in retrapping in the case of the MCSA. While in the high-field region, the gyro-radius of the particle is small. When the low-field region is reached, the particle moves in a straight-line path along the vector direction it has at the edge of the null region. Since the phase of the gyro motion of the particles is random at this point, the vector direction they assume is crossing the null is random. The result then can be viewed, as a random, collisionless, scattering process. Particles that stream along the axis and enter a neighboring SIEC scatter in this way and become confined in the neighboring SIEC (termed axial-loss "re-trapping"). This re-trapping greatly increases the confinement time of a MCSA fusion device. This process is results in a linear scaling of confinement time with the number of units in the SIEC array. Therefore, verifying axial-loss retrapping would be a key objective of early verification experiments.

The experimental verification of the KAM re-trapping effect in the MCSA magnetic field could be carried out simultaneously with the three-step verification of radial loss recirculation experiment. Knowledge of the density within each unit, combined with the known electron (or plasma) injection rate, provides a means of estimating the confinement time of the system for both a single unit and for two units operating in series. Comparison of these measurements with a model for MCSA confinement would provide a test of our understanding of this key effect. A similar study would be done using the plasma gun source. 


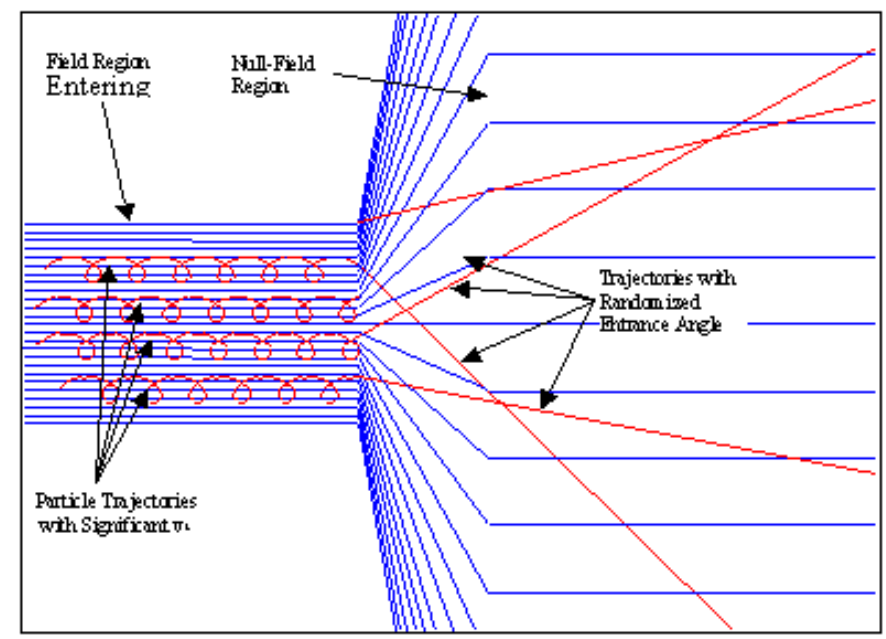

FIGURE 5. Diagram of Direction Randomization (KAM effect) Due to Magnetic Field Null Region in SIEC.

\section{SUMMARY}

If the Physics principles are demonstrated successfully, the MCSA Fusion Power plant will offer numerous advantages for space propulsion. The non-Maxwellian plasma involved is well suited for operation with $\mathrm{D}-\mathrm{He}^{3}$ fuel that provides much of the energy output in the form of very energetic 14-MeV protons. Proton energy can be employed for either direct conversion to electricity or directed thrust (Miley, 2003). As demonstrated in the Fusion Ship II design study, the MCSA power plant is especially well suited for manned deep space missions. In this paper we described the basic features of the MCSA system including recirculation from escaping particles from one unit into the neighboring one where retrapping occurs. These physical principles have not yet been demonstrated experimentally, but as described here, initial tests could be done with a modest small experiment and stepped up in size to eventually demonstrate a full-scale prototype power unit.

\section{ACKNOWLEDGMENTS}

This work was supported under NASA grant: NASA NAS 8-02012. Important contributions by the other workers in the fusion studies lab, especially Linchun Wu, Huyng Jin Kim, and Yang Yang are gratefully acknowledged.

\section{REFERENCES}

Barnes, D.C, Nebel, R.A, and Tiouririne, T.N., "Alternate Fusion; Continous Intertial Confinement," Plasma Phys. Controlled Fusion, 35, 939 (1998).

Burton, R., Momota, H., Richardson, N., Shaban, Y., and Miley, G.H., "Fusion Ship II- A Fast Manned Interplanetary Space Vehicle Using Inertial Electrostatic Fusion," Space Technology and Applications International Forum - (STAIF-2003) edited by M.S. El-Genk, American Institute of Physics, Conference Proceedings 654, p. 553 - 562, (2003).

Bussard, R., "Some Physics Considerations of Magnetic Inertial-Electrostatic Confinement: A New Concept for Spherical Converging-Flow Fusion," Fusion Technology, Vol. 19, No. 2, 273-293 (1991).

Miley, G.H., Momota, H., Stubbers, R., Wu, L., Kim, H.J., and Webber, J., "Experimental Simulation of a Proton Collimator for a Fusion Space Thruster" NASA/MSFC/JPL/UAH 14 ${ }^{\text {th }}$ Annual Advanced Space Propulsion Workshop (ASPW 2003), Huntsville, AL, April 15 - 17, (2003).

Momota, H., Ishida, A., Kohzaki, Y., Miley, G.H., Ohi, S., Ohnishi, M., Sato, K., Steinhauer, L.C., Tomita, Y., and Tuszewski, M., "Conceptual Design of the D-3He Reactor ARTEMIS," Fusion Technology, 21, 2307, (1992).

Momota, H., Miley, G.H., and Nadler, J., "Direct Energy Conversion for IEC Fusion for Space Applications," Proceedings $36^{\text {th }}$ AIAA/ASME/SAE/ASEE Joint Propulsion Conference and Exhibit, Huntsville, AL, July (2000).

Momota, H., Tomita, Y., Ishikawa, M., and Yasaka, Y., "A Traveling Wave Direct Converter for D-3He Fueled Fusion Reactor", Fusion Technology, 35, 60, (1999).

Takahashi, T., Tomita, Y., Momota, H., and Shabrov, N.V., "Collisionless pitch angle scattering of plasma ions at the edge region of a field-reversed configuration" Plasma Physics, 4 (1997) p. 4301 